\title{
Stop press!!!
}

It gives me great pleasure to announce that Springer publishers have just been informed that Forensic Science Medicine and Pathology will soon have an ISI rating. This, in conjunction with rapid e-publishing of accepted manuscripts and registration with Medline, PubMed and SCOPUS, will ensure the widest dissemination of papers published in the journal.

To initiate this new era for the journal, three new sections will be commencing.

The first is a section on Continuing Medical Education kindly provided to us by a series of authors through Prof. Burkhard Madea. The intention of this section is to have up-to-date reviews of particular topics of forensic importance, followed by a series of questions that can be used for readers in their continuing medical education programs with various colleges. If readers feel that they would like to submit educational reviews in this format, this can be done through our online submission system. Many thanks to Prof. Klaus Püschel for permitting the use of this material from Rechtsmedizin.
The second new section is entitled Images in Forensics and is designed to provide an image of an unusual, unique, characteristic, or confusing macroscopic or microscopic finding with a short description, followed on the next page by the answer. It will be an entertaining way of testing our knowledge of certain conditions. Again submissions are very welcome.

The final new section is entitled Differential Diagnosis and is in the same format as Images in Forensics: a brief description with images to be followed by a short dissertation. The aim of this section is to provide a pithy summary of a particular condition of interest, with a discussion of the possible differential diagnosis. It is hoped that both of these sections will draw out the type of fascinating cases that most forensic pathologists hoard in their bottom drawers.

Prof. Roger Byard, Editor in Chief, FSMP 